\title{
VARIABLE TIME STEPS IN THE SOLUTION OF THE HEAT FLOW EQUATION BY A DIFFERENCE EQUATION
}

\author{
JIM DOUGLAS, JR. AND T. M. GALLIE, JR.
}

1. Introduction. Recent improvements in high speed digital computing machines have resulted in focusing considerable attention on the problem of numerical integration of partial differential equations by means of systems of difference equations. A number of these systems have been proposed and have been proved to possess solutions which converge, upon refinement of the "mesh size," to the solution of the boundary value problem. These systems of difference equations, however, appear invariably to involve constant increments in the independent variables. It would require an exorbitant length of time in most cases to carry out the computations with constant increments throughout since these increments must necessarily be small where the solution is varying rapidly, while it has been observed in practice that the size of these increments may be increased in a manner dependent on the type of difference equation used.

In the case of explicit methods the increments of a single variable may not be changed but increments of all variables must be increased simultaneously. This is frequently inconvenient for programming the computations. Implicit methods are more flexible in this regard in that it will be shown the increments of a single variable can be increased from step to step.

It is our purpose here to propose a system of implicit difference equations with variable time increments and demonstrate its convergence to the solution of a boundary value problem for the heat flow equation. This equation appears in a wide variety of problems of diffusion such as the flow of fluids through porous media, heat conduction, and Brownian motion. The initial and boundary values which we consider, while quite restrictive from a mathematical point of view, cover most cases of physical interest. Some discussion of these initial values will be found in the last section of this report. Both linear and exponential growth of the time steps will be considered and it will be shown that in each case the order of convergence is the same as that in the case of constant time steps.

2. The difference equation and truncation error. We shall obtain an approximation to the solution of the boundary value problem 1954.

Presented to the Society, February 26, 1955; received by the editors December 11 , 


$$
\begin{aligned}
\frac{\partial^{2} u}{\partial x^{2}} & =\frac{\partial u}{\partial t}, & t>0,0<x<1, \\
u(0, t)=u(1, t) & =0, & u(x, 0)=f(x),
\end{aligned}
$$

where $f(x)$ has a convergent Fourier sine series

$$
\sum_{n=1}^{\infty} a_{n} \sin n \pi x=f(x), \quad a_{n}=2 \int_{0}^{1} f(x) \sin n \pi x d x,
$$

with

$$
\sum_{n=1}^{\infty} n^{4}\left|a_{n}\right|<\infty
$$

The approximation will be obtained as the solution of a system of implicit difference equations with variable time increments $\Delta t_{0}$, $\Delta t_{1}, \cdots, \Delta t_{M-1}$.

We shall use the following customary notation. Let $t_{0}=0$, $t_{n}=\sum_{k=0}^{n-1} \Delta t_{k}$ for $n=1,2, \cdots, M$ and $T=t_{M}$. Dividing the $x$-interval $(0,1)$ into $N$ intervals of equal length $\Delta x$, let $x_{i}=i \Delta x$ for $i=0,1$, $\cdots, N$, and denote $\rho\left(i \Delta x, t_{n}\right)$ by $\rho_{i, n}$.

The system of difference equations to be considered is

$$
\begin{gathered}
\Delta_{x}^{2} w_{i, n+1}=\frac{w_{i, n+1}-w_{i n}}{\Delta t_{n}}, \quad n=0, \cdots, M-1, i=1, \cdots, N-1, \\
w_{i 0}=f(i \Delta x), \quad w_{0 n}=w_{N n}=0,
\end{gathered}
$$

where

$$
\Delta_{x}^{2} w_{i n}=\left(w_{i-1, n}-2 w_{i n}+w_{i+1, n}\right) /(\Delta x)^{2} .
$$

Now it is easily seen that

$$
\begin{aligned}
\frac{\partial^{2} u_{i, n+1}}{\partial x^{2}} & =\Delta_{x}^{2} u_{i, n+1}-\frac{1}{12} \frac{\partial^{4} u_{i, n}^{*}}{\partial x^{4}}(\Delta x)^{i} \\
& =\Delta_{x}^{2} u_{i, n+1}-\frac{1}{12} \frac{\partial^{2} u_{i, n}^{*}}{\partial t^{2}}(\Delta x)^{2}
\end{aligned}
$$

and

$$
\frac{\partial u_{i, n+1}}{\partial t}=\frac{u_{i, n+1}-u_{i n}}{\Delta t_{n}}+\frac{1}{2} \frac{\partial^{2} u_{i, n}^{*}}{\partial t^{2}} \Delta t_{n}
$$


where the asterisks indicate mean values as required by Taylor's theorem. Substituting in the differential equation we have

$$
\Delta_{x}^{2} u_{i, n+1}=\frac{u_{i, n+1}-u_{i n}}{\Delta t_{n}}+\left(\frac{(\Delta x)^{2}}{12}+\frac{\Delta t_{n}}{2}\right) \frac{\partial^{2} u_{i, n}^{*}}{\partial t^{2}}
$$

where the last derivative is to be evaluated at some point in the rectangle $x_{i-1}<x<x_{i+1}, \mathrm{t}_{n}<t<t_{n+1}$.

Let $v_{i, n}=u_{i, n}-w_{i, n}$. Then

$$
\begin{aligned}
\Delta_{x}^{2} v_{i, n+1} & =\frac{v_{i, n+1}-v_{i n}}{\Delta t_{n}}+\left(\frac{(\Delta x)^{2}}{12}+\frac{\Delta t_{n}}{2}\right) \frac{\partial^{2} u_{i n}^{*}}{\partial t^{2}}, \\
v_{i, 0} & =v_{0, n}=v_{N, n}=0 .
\end{aligned}
$$

Now it has been shown [2] that if

$$
\Delta^{2} y_{i}-\rho_{i} y_{i}=g_{i}, \quad i=1, \cdots, N-1,
$$

with $y_{0}=y_{N}=0$, then

$$
\max _{i}\left|y_{i}\right| \leqq \max _{i}\left|\frac{g_{i}}{\rho_{i}}\right|,
$$

and hence, if we denote $\max _{i}\left|v_{i, n}\right|$ and $\max _{i}\left|\partial^{2} u_{i n}^{*} / \partial t^{2}\right|$ by $\|v\|_{n}$ and $\left\|\partial^{2} u / \partial t^{2}\right\|_{n}$, respectively,

$$
\begin{aligned}
\|v\|_{n+1} & \leqq \max _{v}\left|-v_{i n}+\Delta t_{n}\left(\frac{(\Delta x)^{2}}{12}+\frac{\Delta t_{n}}{2}\right) \frac{\partial^{2} u_{i n}^{*}}{\partial t^{2}}\right| \\
& \leqq\|v\|_{n}+\Delta t_{n}\left(\frac{(\Delta x)^{2}}{12}+\frac{\Delta t_{n}}{2}\right)\left\|\frac{\partial^{2} u}{\partial t^{2}}\right\|_{n} .
\end{aligned}
$$

Or, since $\|v\|_{0}=0$,

$$
\|v\|_{n} \leqq \sum_{k=0}^{n-1} \Delta t_{n}\left(\frac{(\Delta x)^{2}}{12}+\frac{\Delta t_{n}}{2}\right)\left\|\frac{\partial^{2} u}{\partial t^{2}}\right\|_{n} .
$$

It is well known $[1$, p. 76] that the solution of the boundary value problem is $u(x, t)=\sum_{n=1}^{\infty} a_{n} e^{-n^{2} \pi^{2} t} \sin n \pi x$. Differentiating twice,

$$
\frac{\partial^{2} u}{\partial t^{2}}=\pi^{4} e^{-\pi^{2} t} \sum_{n=1}^{\infty} n^{4} a_{n} e^{-\left(n^{2}-1\right) \pi^{2} t} \sin n \pi x,
$$

so that 


$$
\left|\frac{\partial^{2} u}{\partial t^{2}}\right| \leqq \pi^{4} e^{-\pi^{2} t} \sum_{n=1}^{\infty} n^{4}\left|a_{n}\right|=2 A e^{-\pi^{2} t}
$$

and, hence,

$$
\|v\|_{n} \leqq A \sum_{k=0}^{n-1} \Delta t_{k}\left(\frac{(\Delta x)^{2}}{6}+\Delta t_{k}\right) e^{-\pi^{2} t_{k}} .
$$

It is easy to see that if $\Delta t_{n}$ is identically equal to $(\Delta x)^{2}$ then, as $\Delta x$ approaches zero,

$$
\|v\|_{n}=O\left(M(\Delta x)^{4}\right)=O\left((\Delta x)^{2}\right),
$$

but this choice of $\Delta t_{n}$ is clearly not a practical one for computation. In the two sections which follow it will be shown that $\|v\|_{n}$ $=O\left((\Delta x)^{2}\right)$ even if $\Delta t_{n}$ increases linearly or exponentially with $t$.

3. Linear growth of $\Delta t_{n}$. Suppose

$$
\frac{\Delta t_{k}}{(\Delta x)^{2}}=\alpha+\beta t_{k}
$$

where $\alpha$ and $\beta$ are positive constants. Then, by induction,

$$
t_{k}=\frac{\alpha}{\beta}\left[\left(1+\beta(\Delta x)^{2}\right)^{k}-1\right]
$$

and hence

$$
\Delta t_{k}=\alpha(\Delta x)^{2}\left(1+\beta(\Delta x)^{2}\right)^{k}
$$

so that

$$
\begin{aligned}
\|v\|_{n} \leqq & A \alpha e^{-\alpha \pi^{2} / \beta}(\Delta x)^{4} \sum_{k=0}^{\infty}\left(1+\beta(\Delta x)^{2}\right)^{k}\left[1 / 6+\alpha\left(1+\beta(\Delta x)^{2}\right)^{k}\right\rfloor \\
& \cdot e^{-\alpha \pi^{2}\left(1+\beta(\Delta x)^{2}\right)^{k} \beta} .
\end{aligned}
$$

LEMma. If $a>1$ and $c>0$ then

$$
\sum_{k=0}^{\infty} a^{k} e^{-c a k}<\frac{1}{c e^{c} \log a}+\frac{1}{c e}
$$

and

$$
\sum_{k=0}^{\infty} a^{2 k} e^{-c a k}<\left(1+\frac{1}{c}\right) \frac{1}{c e^{c} \log a}+\frac{4}{e^{2} c^{2}} .
$$

To prove this we note that $\eta e^{-c \eta}$ increases monotonically to $1 / c e$ 
and then decreases monotonically and that $\eta^{2} e^{-c \eta}$ behaves similarly, its maximum being $4 / c^{2} e^{2}$. Thus the first series is bounded by $\int_{0}^{\infty} a^{s} e^{-c a^{*}} d s+1 / e c$ and the second by $\int_{0}^{\infty} a^{2 s} e^{-c a^{a}} d s+4 / e^{2} c^{2}$. Making the change of variable $\eta=a^{*}$, these integrals are easily evaluated to complete the proof of the lemma.

Applying the lemma we note that in the case at hand $c=\alpha \pi^{2} / \beta$, which is a constant, and $a=\left(1+\beta(\Delta x)^{2}\right)$ so that

$$
1 / \log a=O\left(1 /(\Delta x)^{2}\right)
$$

as $\Delta x$ approaches zero, and thus the sum in our estimate of $\|v\|_{n}$ is $O\left(1 /(\Delta x)^{2}\right)$ or

$$
\|v\|_{n}=O\left((\Delta x)^{2}\right) \text {. }
$$

4. Exponential growth of $\Delta t_{n}$. Suppose

$$
\frac{\Delta t_{k}}{(\Delta x)^{2}}=e^{\pi^{2} t_{k} / 2}
$$

Then,

$$
\begin{aligned}
\|v\|_{n} & \leqq A(\Delta x)^{4} \sum_{k=0}^{M-1}\left[\frac{1}{6} \frac{\Delta t_{k}}{(\Delta x)^{2}}+\left(\frac{\Delta t_{k}}{(\Delta x)^{2}}\right)^{2}\right] e^{-\pi-t_{k}} \\
& =A(\Delta x)^{4} \sum_{k=0}^{M-1}\left(\frac{1}{6} e^{-\pi^{2} t_{k} / 2}+1\right) \\
& \leqq \frac{7}{6} A M(\Delta x)^{4}
\end{aligned}
$$

Now, for $s \geqq 0, e^{s} \geqq 1+s$, and hence

$$
\begin{aligned}
t_{k+1} & =t_{k}+\Delta t_{k} \geqq t_{k}+(\Delta x)^{2}\left(1+\frac{\pi^{2}}{2} t_{k}\right) \\
& =\left(1+\frac{\pi^{2}}{2}(\Delta x)^{2}\right) t_{k}+(\Delta x)^{2}
\end{aligned}
$$

so that, by induction,

$$
t_{k} \geqq \frac{2}{\pi^{2}}\left[\left(1+\frac{\pi^{2}}{2}(\Delta x)^{2}\right)^{k}-1\right] ;
$$

consequently,

$$
T=t_{M} \geqq \frac{2}{\pi^{2}}\left[\left(1+\frac{\pi^{2}}{2}(\Delta x)^{2}\right)^{M}-1\right]>2 M(\Delta x)^{2} .
$$


Thus,

$$
M=O\left((\Delta x)^{-2}\right)
$$

and, hence,

$$
\|v\|_{n}=O\left((\Delta x)^{2}\right) .
$$

The proof given above is applicable and in fact easier in the case

$$
\Delta t_{k} /(\Delta x)^{2}=e^{\alpha t_{k}}
$$

where

$$
0<\alpha<\pi^{2} / 2 \text {. }
$$

5. Remarks on the initial condition. It will be observed that our hypotheses on the function $f(x)$, that is to say on the initial condition of the solution $u(x, t)$, do not include the possibility $f(x)=$ const. For the Fourier sine series for $f(x)=1$ is

$$
\frac{4}{\pi} \sum_{n=1}^{\infty} \frac{\sin (2 n-1) \pi x}{2 n-1}
$$

and these coefficients fail to satisfy our requirement that

$$
\sum_{n=1}^{\infty} n^{4}\left|a_{n}\right|<\infty
$$

It seems intuitively certain, therefore, that our hypotheses are too stringent.

A number of sufficient conditions can be given on $f(x)$ so that its Fourier series satisfy our requirements. If $f(x)$ has six continuous derivatives and $f(0)=f(1)=0$, the latter being the property $f(x)=1$ fails to possess, then

$$
a_{n}=O\left(1 / n^{5}\right)
$$

as may be seen by integrating $\int_{0}^{1} f(x) \sin n \pi x d x$ by parts. Much less than this is required, however. For instance, it is enough that $f(0)$ $=f(1)=0$ and $f^{(4)}(x)$ satisfy the Lipschitz condition of order $\alpha$

$$
\left|f\left(x_{1}\right)-f\left(x_{2}\right)\right| /\left|x_{1}-x_{2}\right|^{\alpha}=O(1)
$$

for $0 \leq x_{1}, x_{2} \leq 1$ where $\alpha>1 / 2$. For then the Fourier series for $f^{(4)}(x)$ is obtained from that for $f(x)$ by differentiating term by term (this is again seen by integrating by parts) and the former series converges absolutely $\left[3\right.$, p. 135] and hence $\left[3\right.$, p. 131] $\sum_{n=1}^{\infty} n^{4}\left|a_{n}\right|$ converges. Or, if $f^{(4)}(x)$ is of bounded variation, it need merely satisfy a Lip- 
schitz condition of any positive order [3, p. 136], and more general conditions on $f^{(4)}(x)$ are known $[3$, p. 143].

\section{BiBLIOGRAPHY} 1947

1. H. S. Carslaw and J. C. Jaeger, Conduction of heat in solids, Oxford, Clarendon,

2. J. Douglas, On the numerical integration of quasi-linear parabolic differential equations, to appear in the Pacific Journal of Mathematics.

3. Antoni Zygmund, Trigonometrical series, Warsaw-Lwow, 1935.

Humble Oil and Refining Company and

DUKE UNIVERSITY.

\section{ON $n$-POINT EXPANSIONS OF ENTIRE FUNCTIONS}

\section{R. C. $\mathrm{BUCK}^{1}$}

In a recent paper [5], A. J. MacIntyre has studied the representation of entire functions of exponential type in certain $n$-point expansions associated with the names Lidstone, Whittaker, Gontcharoff, and Poritsky. In this note, I shall show that the kernel expansion method which I have previously used to discuss general expansion theorems is also applicable to the cases which he has studied.

The general class of expansion formulae that concern us arise from the problem of expressing a function $f$ in terms of the values of a sequence of its derivatives, computed at a finite set of points. Let $\left\{L_{n}\right\}$ be a sequence of functionals defined on the class $K$ of functions of exponential type by

$$
L_{p k+j(f)}=f^{\left(p k+r_{j}\right)}\left(\beta_{j}\right) \quad k=0,1, \cdots ; j=0,1, \cdots, p-1 .
$$

This sequence is specified by the set of integers $\left\{r_{0}, r_{1}, \cdots, r_{p-1}\right\}$ and the set of complex numbers $\left\{\beta_{0}, \beta_{1}, \cdots, \beta_{p-1}\right\}$. The Lidstone set [10] arise from the choice $p=2, r_{0}=r_{1}=0, \beta_{0}=0, \beta_{1}=1$. The generalization introduced by Poritsky [7] corresponds to $r_{j}=0$ for $j=0$, $1, \cdots, p-1$ with arbitrary $\beta_{j}$. The Whittaker two-point set [10] arises when $p=2, r_{0}=0, r_{1}=1, \beta_{0}=1, \beta_{1}=0$, and the periodic Gontcharoff set corresponds to $r_{j}=j$ with arbitrary $\beta_{j}[4]$.

The general problem is to discover to what extent knowledge of the values $L_{n}(f)$ for $n=0,1, \cdots$ determine $f$. If $f$ is of exponential

Received by the editors June 3, 1954.

1 Supported in part by the Office of Ordnance Research. 